\title{
Mechanical Properties and Chemical Stability of Bathroom Wall Composites Manufactured from Recycled Polyethylene Terephthalate (PET) Mixed with Cocoa Hulls Powder
}

\author{
A. Tom¹, P.N. Djomou Djonga ${ }^{2, *}$, H.G. Valery³, S. Kamdoum Noukelack ${ }^{4}$ \\ ${ }^{1}$ Department of Energy Engineering, University Institute of Technology, University of Ngaoundere, PO Box 455 Ngaoundere, Cameroon.
} ${ }^{2}$ Department of Chemistry, Faculty of Science, University of Maroua, P.O. Box 46 Maroua, Cameroon.

${ }^{3}$ Department of Textile \& Leather Engineering, National Advanced School of Engineering of Maroua, University of Maroua, P.O. Box 46 Maroua, Cameroon. ${ }^{4}$ Nanosciences African Network, Material Research Departmt, iThemba LABS-National Research Foundation, PO Box 722, Western Cape, South Africa.

\section{A R T I C LEDETA I L S}

Article history:

Received 08 November 2021

Accepted 25 December 2021

Available online 31 December 2021

\section{Keywords:}

Chemical Stability

Cocoa Hulls Powder

Composites

Mechanical Properties

PET

\begin{abstract}
A B S T R A C T
The need to protect our environment by eliminating plastic waste as much as possible and by recycling waste from agricultural residue, has led us to formulate composites based on polyethylene terephthalate (PET) loaded with powder from the cocoa shell. The cocoa hulls were prior treated with organosolv process to improve the fiber-matrix interaction. This research is aimed at manufacturing composite wall tiles from recycled PET reinforced with cocoa hull powder (CCP). The composites were manufactured by the melt-mixing method followed by compression molding. The mechanical, physico-chemical properties and the stability to environmental conditions were evaluated. The results showed that the incorporation of cocoa powder at a content of 20-30\% in the matrix consisting of PET gave rise to a composite material with good physico-mechanical and chemical properties suitable for use in several sectors. In the construction industry, in particular as wall covering as a replacement for tiles, these $\mathrm{x}$ from an economic point of view cost less and compared to clays which consumes enormous amount of energy for the elaboration of ceramics. The study showed that the optimum powder weight proportion for the optimal properties of the composite were achieved at $30 \%$ powder weight proportion. The maximum tensile strength of $60.3 \mathrm{MPa}$, flexural strength of $19.5 \mathrm{MPa}$, impact strength of $10.3 \mathrm{MPa}$ and water absorption $1.34 \%$ were obtained. Water absorption of the tiles increased with the cocoa powder weight. Compare to the ceramic tile this value of water absorption test is in range and show that this composite tile is suitable for use as bathroom tile.
\end{abstract}

\section{Introduction}

Plastic consumption and its latter disposal have become a problem due to the high volume of waste and the huge environmental impact they have, not only for the human population, but also for ecological systems [1, 24]. Plastic is a versatile material with wide applications. However, it is a material that people do not consume correctly as there are no perceived dimensions on the environmental damage that its use entails $[1,5,6]$. Per capita plastic consumption continues to rise and remains high in highincome countries, despite obvious contributions to the global issue of plastic pollution $[1,7]$. In 2015, the World Bank concluded that if waste generation maintains the same dynamic without adequate actions to improve reuse, unsustainable use, and production, it will have become a health emergency issue in most countries. This is in addition to high greenhouse gas emissions by 2030. Our planet is not capable of digesting the plastic waste generated daily and this will continue to happen. In Cameroun precisely in Yaoundé and Douala town, each person consumes an average of $2 \mathrm{~kg}$ of plastic per month, $24 \mathrm{~kg}$ per year, this means 1250 Mt per year in those town [8]. The propagation of plastic waste in the environment constitutes a serious threat to public health because it contains in their structures pollutants and heavy metals which have enormous consequences on the health of living beings [9-11]. With the increase in the volume of waste in our cities, it is more than urgent to consider means of reducing these volumes. As a result, while most of the plastic available today is made from non-biodegradable sources, landfilling using plastic would mean burying the harmful material for a period of time until it naturally degrades. However, their degradation rate and bulky nature create enormous environmental risks. In addition, the mass of plastic waste can hinder the movement of groundwater [12]. Hence the need to give new life to the waste which would constitute a raw

material for the construction industry of building materials. Plastic waste can be reused in several sectors of life. Several processes are used. We will cite thermochemical and thermal processes. A very interesting way to dispose of plastic waste is mechanical recycling, which consists of collection, shredding and granulation followed by its reintroduction into the manufacture of other plastic products [13]. Given the soaring costs of construction materials, the ambient unemployment that many young people suffer, this valuation sector can create jobs and reduce poverty which results in the valuation of local raw materials as construction materials. In terms of civil engineering application, recycling of plastic waste as cementitious based materials. This is due to its economic and ecological advantages which can substitute or replace certain portion of aggregate in concrete mix. Besides, some alteration method of plastic waste could be ideal candidate as lightweight concrete pavement with low strength application. As such, there are already a lot of published reports that have been studied concerning the ability of plastic waste mixed together with cementitious based mixtures such as polyethylene terephthalate (PET) bottle, polyvinyl chloride (PVC) pipe, high density polyethylene (HDPE), spent plastic waste, expanded polystyrene foam (EPS), glass reinforced plastic (GRP), polycarbonate, thermoplastic recycled polystyrene, polypropylene fiber as an aggregate, or mixture in the manufacturing of concrete [9]. Reused plastics can be used as reinforcement with natural or synthetic textile fibers in the manufacture of composite materials, the main application of which is the coating of surfaces, walls replacing tiles. Interest in natural fiber reinforced composites (NFRC) is increasing due to their properties of biodegradability, non-carcinogenicity, profitability, and respect for the environment, absence of health risks, easy collection and regional availability. They are also a renewable resource, thus offering a better solution for the sustainability of supply [13]. The NFRC versatile characteristics make it suitable for automobiles, railway coach, building construction, wall partition, cabinets, furniture, and packaging manufacture [6]. NFRC are viable because of the wide availability of natural fibers and agriculture by-product fibers which can be used as

- 
reinforcements $[13,14]$. Researchers have been working round the clock to find new sources of natural fibers that possess comparable physical and mechanical properties to synthetic fibers to be used in reinforcing of composites [13, 15]. Indeed, polymers based on synthetic fibers as reinforcements are expensive and have harmful consequences on the environment. Despite the advantages, natural powder has a high-water absorption capacity, hydrophilic in nature, which makes it difficult to manufacture composites. The powder swell and soften in contact with humidity and consequently absorb water, which contributes to the reduction of their mechanical properties while its hydrophilic character affects the dispersion of the fibers within the matrix phase. To improve the interaction between the powder and the polymer matrix, natural fibers must be physically or chemically modified to increase their reactivity and their physico-mechanical properties. Much work has been done on composites reinforced with natural fibers using fibers such as kenaf, palm oil, bamboo, jute, sisal, coconut and pineapple fibers. But there is hardly any work in the literature that uses cocoa shell powder as a filler. Cameroon is at the forefront of the production and exportation of certain cash crops, such as cocoa (5th largest producer in the world), bananas, pineapples, etc. It has embarked on a process of modernizing its agriculture, and is currently implementing so-called second-generation agriculture, which aims to boost national agricultural production. This generates a lot of agricultural waste. Thus in 2013, around 700,368 tonnes of cocoa hulls were produced and around 2,900,000 tonnes will be produced by 2022 [16]. Only a small part has been used as fertilizer and animal feed [17] which poses environmental problems, because these cocoa shells therefore pollute the soil and the rivers. This research aims to use the powder from the cocoa shell to strengthen recycled PET. The tensile strength, bending, water absorption, resistance to acidic and basic conditions will be evaluated in order to measure the physicochemical and mechanical properties of composites.

\section{Experimental Methods}

\subsection{Materials}

The raw material used in this work consists mainly of waste plastics (PET) used as a binder and cocoa hulls powder. The waste plastic samples were taken in the Center region of Yaoundé, Cameroon. Plastic waste was collected in the Mendong area of Yaoundé. Table 1 gives the physical and mechanical characteristics of collected plastics (PET).

Table 1 Physical, mechanical and thermal characteristics of PETs

\begin{tabular}{lll}
\hline Properties & & PET \\
\hline Absorption rate $(\%)$ & 0.0016 \\
density & 0.93 \\
Resistance to traction $(\mathrm{MPa})$ & 24 \\
Mass volume & $\left(\mathrm{kg} / \mathrm{m}^{3}\right)$ & 1395 \\
Latent heat of & $\mathrm{Fusion}(\mathrm{J} / \mathrm{g})$ & 115 \\
Thermal Capacity & $\mathrm{J} / \mathrm{kg}^{\circ} \mathrm{C}$ & 1090 \\
\hline
\end{tabular}

\subsection{Procedure for Obtaining Cocoa Shell Powder}

After podding, the cockles were cut up and collected in a polythene bag transported to the laboratory. Then dried in a Heraeus brand oven at 45 ${ }^{\circ} \mathrm{C}$ until a constant mass is obtained. The dried slices were crushed using a mechanical grinder and sieved with a $1 \mathrm{~mm}$ sieve. The powders obtained were packaged in plastic bags and stored at room temperature $\left(25 \pm 2{ }^{\circ} \mathrm{C}\right)$ until use.

\subsection{Physicochemical Characterization of Cocoa Shell Powder}

\subsubsection{Water and Dry Matter Content}

The water content was determined using the method described by AFNOR (1982) reported by Bachmann et al. [18]. A mass of the fresh sample is dried at $105^{\circ} \mathrm{C}$ in an oven for 24 hours until a constant weight is obtained.

\subsubsection{Crude Fiber Content}

The determination of the crude fiber content in our powders was determined using performed according to the method described by treating the sample to the boil with sulfuric acid followed by soda. The residue obtained is then dried, calcined and weighed [19].

\subsubsection{Lignin Content}

The lignin content was determined by the Klason method reported by Monties [20]. This so-called Klason lignin method uses the insolubility https://doi.org/10.30799/jacs.243.21070402 property of lignin in a concentrated acidic medium that hydrolyzes and dissolves all other constituents. Its objective is to obtain an essentially woody residue.

\subsubsection{Reducing Sugars Content}

The reducing sugars content was determined using the DNS method described by Fischer et al. (1961). It begins with an extraction of sugars in a hot acidic medium. In a hot alkaline medium, DNS reacts with reducing sugars and changes from its yellow oxidized form to its orange reduced form with a maximum absorption at $530 \mathrm{~nm}$.

\subsubsection{Determination of Mass Loss}

The difference between the mass of the sample before and after the pretreatment represents the mass loss. The sample is dried at $105^{\circ} \mathrm{C}$ before and after pretreatment until a constant mass is obtained.

\subsubsection{Pretreatment of Hulls by the Organosolv Process}

It consists of a batch reactor with a total volume of $100 \mathrm{~mL}$ with the useful volume being $80 \mathrm{~mL}$. The experimental protocol was inspired from that described by Nanfack et al. [21]. A mass of $4 \mathrm{~g}$ of cocoa shell is mixed with $80 \mathrm{~mL}$ of the pretreatment solvent (ethanol-water mixture $52: 48 \mathrm{v} / \mathrm{v}$ ) and loaded into the reactor. The latter is tightly sealed using silicone glue to ensure it is watertight. It is then put in an oven preheated to $200{ }^{\circ} \mathrm{C}$. The treatment is stopped by cooling under running water to room temperature. After cooling, the two solid and liquid phases are separated by filtration. The cocoa shell powder thus treated is dried and stored in a glass jar for later use.

\subsection{Manufacturing Method of Composite Materials}

In this study, fine-sized plastic waste is obtained after shredding with a chisel. Plastic waste is then washed with water, dried at room temperature $\left(25{ }^{\circ} \mathrm{C}\right)$ for three days. Composite for PET and cocoa powder: the appropriate with of cocoa powder was added to melt PET in a Haack Rheomix at $225^{\circ} \mathrm{C}$. The mixture weighing $200 \mathrm{~g}$ were processed for 10 minutes. The mixture obtained was then hot press at $225^{\circ} \mathrm{C}$ for 5 minutes at a pressure of $15 \mathrm{MPa}$ and cooled under pressure. The temperature of $225{ }^{\circ} \mathrm{C}$ was used because it does not affect the powder properties. Composite tiles of size $200 \times 150 \times 8 \mathrm{~mm}$ were prepared using a closed mold. The mold was polished with a release agent to avoid PET from sticking to it. The process involved melting of the shredded PET, adding predetermined proportion of the cocoa powder, melt-mixing thoroughly to form a homogeneous viscous solution, and placing it into the prepared mold. Finally, the mold was closed and the samples were cooled down to room temperature under $15 \mathrm{MPa}$ pressure for $30 \mathrm{~min}$. The specimens of the tiles produced were shaped by sandpaper and used for testing. Sheets of $3 \mathrm{~mm}$ thickness were obtained. The materials were made at different powder concentrations (Table 2).

Table 2 Formulation of the different composites according to the addition of the powder

\begin{tabular}{lllllll} 
powder & & & & \\
\hline of CCP & 0 & 10 & 20 & 30 & 40 & 50 \\
\hline$\%$ of PET & 100 & 90 & 80 & 70 & 60 & 50 \\
\hline
\end{tabular}

\subsection{Mechanical Properties and Chemical Stability of Composite}

\subsubsection{Tensile Test}

The determination of the tensile tests was performed on the Instron 5567 at a crosshead speed of $50 \mathrm{~mm} / \mathrm{min}$. Samples were prepared for this test according to the ASTM D638 test standard. At the start of the tensile test, the test piece lengthens and the resistance of the test piece increases, which was detected using a load cell. This value was recorded until the sample ruptured, and five samples were tested [13].

\subsubsection{Flexural Test}

Flexural strength is the combination of tensile strength and compressive strength. The tests were done on a universal testing machine. The specimens were prepared according to the ASTM D790 test standard with dimension $200 \times 30 \times 8 \mathrm{~mm}$. The specimens were tested on a support span of $130 \mathrm{~mm}$ as per the standard [13].

\subsubsection{Impact Test}

Charpy impact tests on unnotched specimens were performed using a pendulum impact testing machine JBS-300 N model. The test specimens were prepared according to the ASTM D6110-18 test standard with a dimension of $50 \mathrm{~mm}$ long, and a cross-sectional area of $24 \mathrm{~mm}^{2}$. Five specimens were tested and an average value was reported [13]. 


\subsubsection{Water Absorption}

Water absorption was carried out on cylindrical fired test specimens according to ASTM-C373 standard [22] where ceramic samples were weighed and immersed in a beaker containing distilled water and the ensemble boiled for 2 hours, and allowed to cool for 24 hours at room temperature. The specimens were then wiped with a paper towel and weighed.

\subsubsection{Chemical Stability}

The chemical stability will consist in soaking our composite tiles in an acid solution and a base solution with respective $\mathrm{pH}$ of 3 and 12 in order to evaluate the loss of mass over a period of 7 days.

\section{Results and Discussion}

\subsection{Characterization of Cocoa Powder Hulls}

\subsubsection{Chemical Composition of Cocoa Powder Hulls}

The cocoa hulls powder studied has a dry matter content of $88.1 \%$ and an organic matter content of $92.1 \%$. This high organic matter content shows that cocoa hulls can be an important source of nutrients for microorganisms in anaerobic digestion, this can explain why it's used as fertilizer. These results are close to those of Daud et al. [23]. On the other hand, these values are slightly higher than those of Vriesmann et al. [24] which had $85.9 \%$ for dry matter and $87.7 \%$ for organic matter. In addition, Ba et al. [25] had high values, i.e., a dry matter content of $91.5 \%$ and $93.3 \%$ for organic matter. As regards the lignocellulosic composition, there are $40.1 \%$ crude fibers, $35.3 \%$ lignin and $41.4 \%$ cellulose. These results are in line with those of Daud et al. [23] who for these same compounds had $42.22 \%, 38 \%$ and $43.13 \%$ respectively. In addition, these contents are higher than that of Ba et al. [25] whose work revealed a crude fiber content of $36.6 \%$ against $21.4 \%$ of lignin. Such lignin (between 10 and $35 \%$ ) and cellulose (between 20 and 50\%) contents immediately make cocoa shells a lignocellulosic biomass according to Balakrishnan et al. [26]. All these results demonstrate the variability in the composition of cocoa shells. This can be explained by differences in the soil composition of the place of origin, the difference in the degree of maturity as well as the harvest period. But the fact remains that cocoa powder constitute a promising source of loaders that can be used as reinforcement. The results are given in Table 3 below.

Table 3 Chemical composition of cocoa powder hulls

\begin{tabular}{ll}
\hline Constituents & Before pretreatment \\
\hline Dry matter content $\left(\mathrm{g} / 100^{*} \mathrm{~g}\right)$ & $88.1 \pm 0.1$ \\
Water content $\left(\mathrm{g} / 100^{*} \mathrm{~g}\right)$ & $11.9 \pm 0.1$ \\
organic matter content $\left(\mathrm{g} / 100^{+} \mathrm{g}\right)$ & $92.1 \pm 0.2$ \\
Ash content $\left(\mathrm{g} / 100^{+} \mathrm{g}\right)$ & $7.9 \pm 0.2$ \\
Reducing sugar content $\left(\mathrm{g} / 100^{*} \mathrm{~g}\right)$ & $0.020 \pm 0.003$ \\
Crude fiber content $\left(\mathrm{g} / 100^{+} \mathrm{g}\right)$ & $40.1 \pm 1.2$ \\
Lignin content $\left(\mathrm{g} / 100^{+} \mathrm{g}\right)$ & $35.3 \pm 1.8$ \\
Cellulose content $\left(\mathrm{g} / 100^{+} \mathrm{g}\right)$ & $41.4 \pm 0.3$ \\
\hline
\end{tabular}

+ Dry base, * Wet base

\subsubsection{Thermal Analysis}

The ATG-DTG curves of the cocoa hulls powder are shown in Fig. 1. Thermogravimetric analysis (ATG) (in red) provides the loss of mass of the material during thermal degradation. The heat treatment phenomenon is described by Verma et al. [27]. The second derivative of thermogravimetric analysis (DTG) (in blue) gives the degradation temperatures of the hulls as defined by Balakrishnan et al. [26].

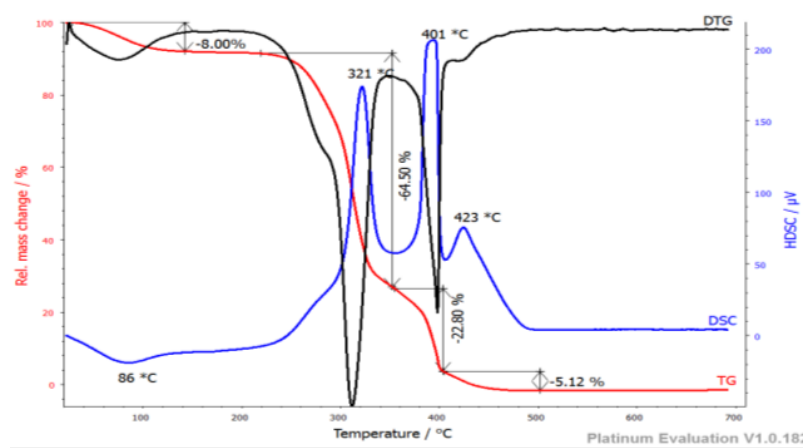

Fig. 1 Thermogravimetric and differential scanning calorimetry of raw cocoa powder hulls

https://doi.org/10.30799/jacs.243.21070402
Observation of the curve shows that around $440{ }^{\circ} \mathrm{C}$, the biomass has been completely consumed. Fig. 1 shows four main phases of mass loss. The first mass loss of $8.00 \%$ by weight is located between 40 to $100{ }^{\circ} \mathrm{C}$ and has a peak around $86^{\circ} \mathrm{C}$; it corresponds to the evaporation of the water present in the hulls. The second loss of mass correspond to mass loss of $64.50 \%$ is observed between 300 and $400{ }^{\circ} \mathrm{C}$, with a DTG maximum at 321 ${ }^{\circ} \mathrm{C}$. It is assigned to the degradation of polysaccharides (hemicelluloses and cellulose) in the fiber of cocoa hulls [27]. The third loss of 22,60\% observed between 321 and $401{ }^{\circ} \mathrm{C}$ would be due to the degradation of cellulose; in fact, cellulose would degrade around $401{ }^{\circ} \mathrm{C}$. Between $401{ }^{\circ} \mathrm{C}$ and $423^{\circ} \mathrm{C}$, a loss of $5.12 \%$ is observed having a peak around $423^{\circ} \mathrm{C}$, which would be due to the degradation of the lignin at $423^{\circ} \mathrm{C}[27]$.

It was noted that these results are in agreement with the chemical composition of cocoa hulls: the major constituents of which are cellulose, hemicellulose and lignin; they degrade with a strong loss of mass [28]. In addition, hemicelluloses decompose at low temperatures because their molecular chains are short. In addition to their low molecular mass, they have fewer regular structures in their chains [28]. The curve of Fig. 1 shows four thermal phenomena for cocoa hulls. One endothermic peak observed at lower temperature around $86^{\circ} \mathrm{C}$ and three exothermic peaks range between $321{ }^{\circ} \mathrm{C}, 401{ }^{\circ} \mathrm{C}$ and $423{ }^{\circ} \mathrm{C}$. The first thermal incident was attributed to evaporation of water molecules from fibers, the second third and fourth respectively represent the degradation of holo-celluloses, celluloses and lignin from the coffee hulls [28]. Table 4 give the different types of thermal phenomena that occur during the thermal analysis of the sample.

Table 4 Types of thermal phenomena

\begin{tabular}{lllll}
\hline & $\begin{array}{l}\text { Temperature } \\
\left({ }^{\circ} \mathrm{C}\right)\end{array}$ & $\begin{array}{l}\text { Partial loss of } \\
\text { mass }(\%)\end{array}$ & $\begin{array}{l}\text { Total loss of } \\
\text { mass }(\%)\end{array}$ & Type of reaction \\
\hline \multirow{3}{*}{ Powder of } & 86 & 8 & & Endothermic \\
cocoa hulls & 321 & 64.50 & 100 & Exothermic \\
& 401 & 22.8 & & Exothermic \\
& 423 & 5.12 & & Exothermic \\
\hline
\end{tabular}

\subsubsection{Fourier Transform Infrared Analysis}

As shown in Fig. 4 and Table 5, the FTIR analysis of cocoa hulls powder, shows different peak intensities. Five main groups of adsorption bands emerge, namely those between $3000-3500 \mathrm{~cm}^{-1}, 3000-2500 \mathrm{~cm}^{-1}, 2000$ $-1500 \mathrm{~cm}^{-1}, 1000-500 \mathrm{~cm}^{-1}$. The wavenumber bands between 3000 $\mathrm{cm}^{-1}$ and $3500 \mathrm{~cm}^{-1}$ are attributed to the vibration of elongation of the linked alcohol $\mathrm{OH}$ group. The wavenumber between $2500 \mathrm{~cm}^{-1}$ and 3000 $\mathrm{cm}^{-1}$ shows the presence of the $\mathrm{CH}$ elongation bond. The adsorption band between $2000 \mathrm{~cm}^{-1}$ and $1400 \mathrm{~cm}^{-1}$ is characteristic of $\mathrm{CO}$ elongation. The absorption band of $1000 \mathrm{~cm}^{-1}$ corresponds to the CC bond.

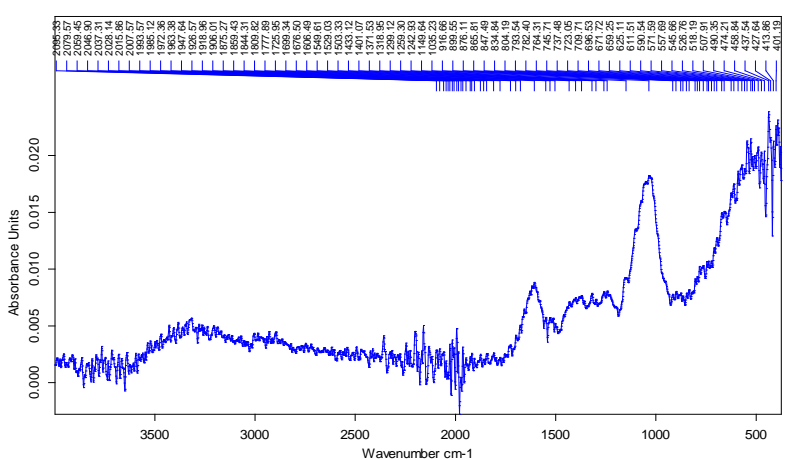

Fig. 2 Infrared of raw cocoa shell powder

Table 5 IR spectrum of cocoa shell powder: The characteristic infrared vibration bands, relating to CCP

\begin{tabular}{ll}
\hline Number of waves $\left(\mathrm{cm}^{-1}\right)$ & attribution \\
\hline 3315 & $\sqrt{ }(\mathrm{OH})$ of lignin and polysaccharides \\
2927 & $\sqrt{\text { as }}(\mathrm{C}-\mathrm{H})$ of $\mathrm{CH}$ and $\mathrm{CH}_{2}$ \\
1733 & $\sqrt{ }(\mathrm{C}=\mathrm{O})$ esters and carboxylic acids (xylans, lignins) \\
1602 & Lignin aromatic skeleton \\
1455 & $\delta_{\text {as }}(\mathrm{C}-\mathrm{H})$ from lignin and xylans \\
1417 & $\delta_{\text {as }}(\mathrm{C}-\mathrm{H})$ polysaccharides \\
1371 & $\delta_{\text {as }}(\mathrm{C}-\mathrm{H})$ of cellulose and hemicelluloses \\
1317 & $\mathrm{CH}$ from cellulose and hemicellulose \\
1234 & $\sqrt{ }(\mathrm{C}-\mathrm{O})$ of methoxy groups of lignin \\
1025 & $\delta(\mathrm{C}-\mathrm{O})$ of polysaccharides $\delta(\mathrm{C}-\mathrm{OH})$ of cellulose \\
896 & $\delta(\mathrm{CH})$ of cellulose \\
600 & $\delta(\mathrm{CO}-\mathrm{H})$ of cellulose \\
\hline
\end{tabular}

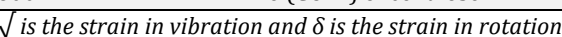




\subsection{Mechanical Characterization of Composites Materials}

Different formulations were carried out with incorporation rates of the cocoa pod powder from 0 to $50 \%$. The following properties, flexural strength, tensile strength, impact strength and water absorption have been used to define the properties of composites. As shown in Table 6, the mechanical properties of composites are given below.

Table 6 Mechanical properties of composite

\begin{tabular}{lllll}
\hline \%CCP & $\begin{array}{l}\text { Tensile } \\
\text { Strength (MPa) }\end{array}$ & $\begin{array}{l}\text { Flexural Strength } \\
(\mathrm{MPa})\end{array}$ & $\begin{array}{l}\text { Impact } \\
\text { Strength (MPa) }\end{array}$ & $\begin{array}{l}\text { Water } \\
\text { Absorption (\%) }\end{array}$ \\
\hline 10 & 55.8 & 10.6 & 8.9 & 1.18 \\
20 & 58.2 & 11.9 & 7.4 & 1.20 \\
30 & 60.3 & 19.5 & 10.3 & 1.34 \\
40 & 56.1 & 12.3 & 7.6 & 1.49 \\
50 & 43.9 & 9.8 & 6.9 & 1.51 \\
\hline
\end{tabular}

\subsubsection{Tensile Strength}

The tensile strength was studied as a function of the rate of incorporation of the cocoa powder into the PET. Table 6 show that the tensile strength of the composite increases with the amount of load introduced $10 \%$ to $30 \%$ and is equivalent to a value of 55.8 to $60.3 \mathrm{MPa}$ respectively, but when the level of $30 \%$ powder is reached, this value drops from 60.3 to $43.9 \mathrm{MPa}$. Observation of the table indicates that the greatest value of the tensile strength is reached after adding $30 \%$ of powder. This high value of the resistance results in a reinforcement of the PET which has become more rigid and can withstand large loads. The small size and their particle size distribution in the composite allows good adhesion and contributes to the densification of the composite. Beyond $30 \%$ of the powder content, the resistance to decrease which would lead to believe that the PET matrix was not sufficient to cross and homogeneously fluidize the surface of the composite creating pores which would contribute to the low resistance to traction.

\subsubsection{Flexural Property}

Analysis of the table reveals that the flexural strength increases with the addition of the powder. The incorporation rate of 10 to $30 \%$ made it possible to go from a value of 10.5 to $19.50(\mathrm{MPa})$. This could be explained by the fact that an increase in the loading rate would lead to the presence of a high rate of loading on a cross section of the composite and therefore increasing the flexural strength. We also note that beyond $30 \%$ of the incorporation rate, there is a decrease in the value of the flexural strength. This drop could be explained by poor dispersion of the powder in the matrix and this observation is confirmed by the tensile strength where we see similar results.

\subsubsection{Impact Strength}

The Table 6 presents the values of the Charpy impact resistance (CIS) of non-notched samples of PET composites with a proportion of cocoa shell powder varying from 10 to $50 \%$. For a load rate below $30 \%$, there is an increase in the CIS which reaches its highest value 10.3 (MPa) at 30\% CCP. Beyond $30 \%$ the CIS decreases, because the addition of more powder creates regions of stress concentrations which require comparatively less energy to initiate a crack; this reduction could also be explained by the fact of the low resistance of the interface between the powder and the matrix. During impact, most of the energy absorbed is used to increase the distance between the load and the die.

\subsubsection{Water Absorption}

As shown in the Table 6 the filler content has a notable effect on water absorption. After $24 \mathrm{~h}$ of immersion in water, the lowest water absorption rate was reached with a load proportion of $10 \%$. The addition of the powder helps to increase the water absorption rate as more lignocellulosic powders are added to the composite, which means that more hydrogen bonds have formed between the water molecules and the $\mathrm{OH}$ group in powders. The same results were observed by Huner et al. who reported that the rate of water absorption increased with increasing fiber content $[29,30]$. This could be explained by the formation of less surface interaction between the matrix and the powder when mixed, resulting in higher water absorption [31].

\subsection{Chemical Stability}

Table 7 shows that after ten days of impregnation in acidic medium and neutral medium, the composite material undergoes a mass loss of $8 \%$ and $2 \%$ respectively; whereas in a basic medium no loss is observed. This result reflects the good chemical stability of the composites under extreme https://doi.org/10.30799/jacs.243.21070402 conditions and shows that the material can indeed be applied as a tile coating for the walls of toilets and showers.

Table 7 Weight loss of specimen in nitric acid and sodium hydroxide solutions (g)

\begin{tabular}{lllllll}
\hline Day & 0 & 2 & 4 & 6 & 8 & 10 \\
\hline $30 \%$ CCP at pH 3.2 & 150 & 143 & 141 & 139 & 139 & 138 \\
$30 \%$ CCP at pH 6.79 & 150 & 148 & 147 & 147 & 147 & 147 \\
$30 \%$ CCP at pH 12.4 & 150 & 151 & 151 & 150 & 150 & 150 \\
\hline
\end{tabular}

\section{Conclusion}

The paper studied the chemical stability and mechanical properties of bathroom wall composites manufactured from recycle polyethylene terephthalate mixed with cocoa hulls powder. Organosolv treatment of cocoa powder hulls enhanced the quality of the cocoa powder hulls by removal of hemicellulose and lignin thereby increased the cellulose composition. The organosolv treatment gave a more thermally stable composite material. The addition of cocoa powder hulls produces an environmentally friendly material. It is clear that cocoa powder hulls have great potential as a filler and reinforcement for composites requiring similar properties to PET. The recycled PET/30\% cocoa hulls powder blend has higher tensile, flexural and impact strength compared to other blends. Recycled PET/30\% cocoa hulls powder blend was also found to have good properties in term of stability. The manufactured composites showed improved mechanical properties with respect to the powder content increase, showing the reinforcement potential of cocoa powder. It is thus possible to consider a prospective industrial use of this agricultural waste, for instance, for the manufacture of bathroom wall tiles in construction industry in Cameroon.

\section{References}

[1] L.A. Sanchez-Echeverri, N.J. Tovar-Perilla, J.G. Suarez-Puentes, J.E. BravoCervera, D.F. Rojas-Parra, Mechanical and market study for sand/recycledplastic cobbles in a medium-size colombian city, Recycl. 6(1) (2021) 17:1-13.

[2] M. Cordier, T. Uehara, How much innovation is needed to protect the ocean from plastic contamination?, Sci. Total Environ. 670 (2019) 789-799.

[3] P.K. Rai, J. Lee, R.J.C. Brown, K.H. Kim, Environmental fate, ecotoxicity biomarkers, and potential health effects of micro- and nano-scale plastic contamination, J. Hazard. Mater. 403 (2021) 123910.

[4] D.R.G. Ribeiro-Brasil, N.R. Torres, A.B. Picanço, D.S. Sousa, V.S. Ribeiro, et al., Contamination of stream fish by plastic waste in the Brazilian Amazon, Environ. Pollut. 266 (2020) 115241.

[5] N.J. Beaumont, M. Aanesen, M.C. Austen, T. Börger, J.R. Clark, et al., Global ecological, social and economic impacts of marine plastic, Mar. Pollut. Bull. 142 (2019) 189-195.

[6] S. Rhein, M. Schmid, Consumers' awareness of plastic packaging: More than just environmental concerns, Resour. Conserv. Recycl. 162 (2020) 105063.

[7] S.J. Barnes, Out of sight, out of mind: Plastic waste exports, psychological distance and consumer plastic purchasing, Glob. Environ. Chang. 58 (2019) 101943.

[8] A.A. Souop Tagne, Modeling of biogas production by the particle swarm method: Application to cocoa hulls, Thesis presented in view of obtaining the Master's degree in Process Engineering, University of Ngaoundéré, Cameroon, 2018.

[9] M.A. Kamaruddin, M.M.A. Abdullah, M.H. Zawawi, M.R.R.A. Zainol, Potential use of plastic waste as construction materials: recent progress and future prospect, IOP Conf. Series: Mater. Sci. Eng. 267 (2017) 012011

[10] N. Saikia, J. de Brito, Use of plastic waste as aggregate in cement mortar and concrete preparation: A review, Constr. Build. Mater. 34 (2012) 385-401.

[11] M.A. Kamaruddin, M.S. Yusoff, N. Ibrahim, M.H. Zawawi, Resource recovery from municipal solid waste by mechanical heat treatment: An opportunity, AIP Conf. Proc. 1835 (2017) 020031.

[12] R. Silva, J. De Brito, R. Dhir, Properties and composition of recycled aggregates from construction and demolition waste suitable for concrete production, Constr. Build. Mater. 65 (2014) 201-217.

[13] N. Gebremedhin, G.K. Rotich, Manufacturing of bathroom wall tile composites from recycled low-density polyethylene reinforced with pineapple leaf fiber, Int. J. Polym. Sci. 2020 (2020) 2732571.

[14] V.P. Cyras, S. Iannace, J.M. Kenny, A. Vázquez, Relationship between processing and properties of biodegradable composites based on PCL/starch matrix and sisal fibers, Polym. Compos. 22(1) (2001) 104-110.

[15] N.M. Barkoula, S.K. Garkhail, T. Peijs, Biodegradable composites based on flax/polyhydroxybutyrate and its copolymer with hydroxyvalerate, Ind. Crop. Prod. 31(1) (2010) 34-42.

[16] FAO, Bioenergy and Food Security Rapid Assessment (BEFS RA): Briquettes, Food and Agriculture Organization, Rome, 2014.

[17] K. Edoh Adabe, E.L. Ngo-Samnick, Cocoa production and processing, Pro-Agro Collection, Douala, Cameroun, 2014.

[18] N. Bachmann, M. Deront, H. Fruteau, C. Holliger, Y. Membrez, A. Wellinger, Optimization of standardized digestibility tests in batch reactors, Federal Office of Energy SFOE Energy Research Program, Bern, Laboratory of Environmental Biotechnology (LBE), 1015 Lausanne, Switzerland, 2011. 
[19] J.P. Wolff, Methods of analysis of corps grass, Azoulay, Paris-France, 1968.

[20] B. Monties, Determination of insoluble lignin in an acid medium: influence of pre-treatment by acid hydrolysis on Klason lignin from wood and straw, Agronomie 4(4) (1984) 387-392.

[21] N.C. Nanfack, Production of biogas from cocoa waste, Master's Thesis in Process Engineering and Engineering, University of Ngaoundéré, Cameroon, 2015.

[22] ASTM-C373, Standard test method for water absorption, Bulk Density, Apparent, Porosity, and Apparent Specific Gravity of Fired White ware Products, C373-88, American standard and testing materials, USA, 1999.

[23] Z. Daud, A.S. Kassi, A.M. Aripin, H. Awang, M.Z. Hatta, Chemical composition and morphological of cocoa pod husks and cassava peels for pulp and paper production, Aust. J. Basic \& Appl. Sci. 7(9) (2013) 406-411.

[24] L.C. Vriesmann, R.D. Amboni, C.L. Petkowicz, Cacao pod husks (Theobroma cacao L.): composition and hot-water-soluble pectins, Ind. Crop. Prod. 34 (2011) 1173-1181.

[25] Y.Y. Bai, L.P. Xiao, Z.J. Shi, R.C. Sun, Structural variation of bamboo lignin before and after ethanol organosolv pretreatment, Int. J. Mol. Sci. 14 (2013) 2139421413.
[26] P. Balakrishnan, M.J. John, L. Pothen, M.S. Sreekala, S. Thomas, Natural fibre and polymer matrix composites and their applications in aerospace engineering, In: Advanced composite materials for aerospace engineering, Woodhead Publishing, Sawston, Cambridge, UK, 2016, pp.365-383.

[27] D. Verma, I. Senal, Natural fiber-reinforced polymer composites, In: Woodhead Publishing Series in Composites Science and Engineering, Biomass Biopolym. Based Mater. Bioenergy, Woodhead Publishing, UK, 2007, pp.103-122.

[28] R. Kumar, M.I. Ul Haq, A. Raina, A. Anand, Industrial applications of natural fibre-reinforced polymer composites-challenges and opportunities, Int. J. Sustain. Eng. 12 (2019) 212-220.

[29] U. Huner, Effect of water absorption on the mechanical properties of flax fiber reinforced epoxy composites, Adv. Sci. Technol. Res. J. 9(26) (2015) 1-6.

[30] W.Z.W. Zahari, R.N.R.L. Badri, H. Ardyananta, D. Kurniawan, F.M. Nor, Mechanical properties and water absorption behavior of polypropylene / ijuk fiber composite by using silane treatment, Procedia Manuf. 2 (2015) 573-578.

[31] H.N. Dhakal, Z.Y. Zhang, M.O.W. Richardson, Effect of water absorption on the mechanical properties of hemp fibre reinforced unsaturated polyester composites, Compos. Sci. Technol. 67(7-8) (2007) 1674-1683. 